therapy, to offer the methodological reference for the Pharmacoeconomic evaluation of the domestic hypertension Intervention.

Methods 1. According to the natural history of hypertension, Markov model was constructed to simulate the dynamic changes of the five states (event free, non-fatal myocardial infarction, non-fatal stroke, natural death and non-natural death) in the hypertension patients who received the two combined treatment, and a 1-year cycle length was chosen. 2. We had applied Markov model using Roll back analysis, Markov cohort simulation and Monte Carlo simulation analysis to project expected life years, the expected qualityadjusted life years and the medical costs in the subsequent $40 \mathrm{yrs}$ of life time for the hypertension patients who had been long-term treated with low dose of amlodipine plus amiloride or telmisartan. Sensitivity analysis were carried out to determine the robustness of our baseline results. 3. On the basis of the published study of China's population-based clinical trials, we had obtained Markov model transition probability between states, health utility values and health care costs in the states through literature review and search statistical data of China.

Results 1 . The baseline Roll back analysis showed that after amlodipine plus amiloride antihypertensive therapy for 40 years, the average cost-effectiveness ratio was 1416 yuan/OALY and 1790 yuan/ OALY respectively. According to Monte Carlo simulation analysis, the average cost-effectiveness ratio of the amlodipine plus amiloride treatment and its 95\% CI were 1173.1 yuan/OALY (95\% CI 1139.43 yuan/OALY to 1174.72 yuan/OALY). 2. The baseline Roll back analysis after amlodipine plus telmisartan antihypertensive therapy for 40 years showed that the average cost-effectiveness ratio was 2252 yuan/LYG and 2334 yuan/OALY respectively. According to Monte Carlo simulation analysis, the average cost-effectiveness ratio of the amlodipine plus telmisartan treatment and its $95 \% \mathrm{CI}$ were 1843.6 yuan/OALY (95\% CI 1, 817.15 yuan/OALY 1850.00 yuan/OALY). 3. Compared with amlodipine plus amiloride antihypertensive therapy, the incremental cost-effectiveness ratio of telmisartan plus amlodipine treatment was 75713 yuan/OALY. The incremental net monetary benefit and the incremental net health benefit were -7480.1 yuan (95\% CI -7804.6 to -7155.6 yuan) and -0.329 OALYs (95\% CI -0.343 OALYs to -0.315 OALYs), which used the 2008 per capita GDP 22698 yuan as the threshold. The probability that the net benefits of the Amlodipine plus amiloride treatment was greater than that of telmisartan plus amlodipine treatment was $94.5 \%$. 4. According to the sensitivity analysis, the change of key parameters in the set range did not affect the model results.

Conclusion 1. Two combination regimens were able to attain significant clinical effectiveness and economic Benefit, however in the case of limited resources, priority should be given to the amlodipine plus amiloride combined antihypertensive therapy as the initial program, in order to obtain better economic benefit. 2. Markov model can be good used for Economic evaluation of blood pressure intervention.

\section{e0589 EFFECTS OF INITIAL THERAPY FOR HYPERTENSION WITH COMBINATION THERAPY REGIMEN AND IMPACT ON RENAL FUNCTION}

doi:10.1136/hrt.2010.208967.589

${ }^{1}$ Haiqiang Sang, ${ }^{2}$ Ming Sun, ${ }^{2}$ Hongyan Zhou. ${ }^{1}$ Zhengzhou People's Hospital; ${ }^{2}$ Xiangya Hospital of Central South University

Objective To evaluate the effects and side effects of initial therapy for hypertension with a combination of Iow-dose amlodipine plus amiroride or low-dose amlodipine plus telmisartan regimen.

Method A total of 302 hypertensive patients were included. Inclusion criteria were: essential hypertension, 50-79 years of age with at least one cardiovascular risk factor and signed consent forms.
Patients were randomly assigned to receive low-dose amlodipine plus amiroride (group A) or low-dose amlodipine plus telmisartan (group B). Blood pressures, side effects, metabolic parameters and renal function indexes were observed during 1-year following-up. All patients will be followed-up for 1 year.

Results After 1-year treatment, mean blood pressure in group $A$ and $B$ were reduced to $(128.1 \pm 10.3) /(76.6 \pm 8.0) \mathrm{mm} \mathrm{Hg}$ and $(131.5 \pm 12.3) /$ $(77.3 \pm 9.2) \mathrm{mm} \mathrm{Hg}$ from $(157.1 \pm 12.0) /(91.1 \pm 9.4) \mathrm{mm} \mathrm{Hg}$ and $(156.4 \pm 13.6) /(91.2 \pm 9.5) \mathrm{mm} \mathrm{Hg}(\mathrm{p}<0.05)$, respectively. Blood pressure control rates reached $87.1 \%$ in group $\mathrm{A}$ and $76.5 \%$ in group $\mathrm{B}$ $(p=0.024)$ the serum uric acid level significantly elevated from $(310.59 \pm 76.32) \mu \mathrm{mol} / \mathrm{l}$ to $(353.71 \pm 76.77) \mu \mathrm{mmol} / \mathrm{l}(\mathrm{p}=0.000)$ after treatment in group $B$. The serum creatinine level decreased from $(85.15 \pm 21.25) \mu \mathrm{mmol} / 1$ to $(82.70 \pm 20.21) \mu \mathrm{mmol} / \mathrm{l}(\mathrm{p}=0.001)$ after treatment in group $\mathrm{B}$.

Conclusion Initial low-dose andodipine-based antihypertensive combination regimens could significantly decrease blood pressure level and achieve satisfactory blood pressure control rate with few side effects. Compatibility of telmisartan may provide renal protection independent of BP lowering in combination regimen. The two combination schemes could markedly improve quality of life in patients with hypertension.

\section{e0590 THE ONE-YEAR PHARMACOECONOMIC EVALUATION OF HYPERTENSION'S TREATMENT BY LOW DOSE AMLODIPINE PLUS TELMISARTAN OR AMLODIPINE PLUS AMIRORIDE REGIMEN}

doi:10.1136/hrt.2010.208967.590

${ }^{1}$ Haiqiang Sang, ${ }^{2}$ Ming Sun, ${ }^{2}$ Hongyan Zhou. 'Zhengzhou People's Hospital; ${ }^{2}$ Xiangya Hospital of Central South University

Objective To evaluate the cost-effectiveness of low-dose amlodipine plus telmisartan (group A) or amlodipine plus amiroride (group B) therapy in hypertensive patients using a decision-tree model with a time horizon of 1 year. To provide evidence on the optimal combination therapy regimens and treatment options in hypertensive patients.

Method Based on the trial data, a cost-effectiveness decision-tree model was developed to assess, about 1 year period, the short-time economic effects, where the total effective rates, Blood pressure control rates and OALYs were estimated as indicators of effectiveness, respectively. Roll back, cost-effectiveness analysis, cost-utility analysis and incremental cost-effectiveness analysis wore adopted in the decision-tree model. Both one-way and two-way sensitivity analysis were carried out to determine the robustness of our baseline results.

Results 1. Expected values: About the average cost per patient needed with a time horizon of 1 year, group A need $¥ 1247$, group B need $¥ 1917$. About the total effective rates and blood pressure control rates, group A reached $87.6 \%$ and $80.9 \%$, group B reached $84.0 \%$ and $70.7 \%$. About the average OALYs per patient gained, group A were 0.046 OALYs and group B were 0.085 OALYs. 2. The one year cost-effectiveness analysis showed that, the cost of effective treatment was $¥ 1389$ and $¥ 2230$ per patient in group A and group $\mathrm{B}$, respectively.At the cost of blood pressure controlled for each case, group A need $¥ 1540$, and group B require $¥ 2712$. At the cost of gain 1 QALY after 1 year therapy, group A need $¥ 26979$, and group B require $¥ 22517$. The estimated ICER for group A vs group B was $¥ 17222$ per OALY gained. 3. The sensitivity analysis results showed that there were no impact of variations in key model inputs on the model.

Conclusion From group decision-making considerations, applying the economic benefit, the initial low-dose amlodipine plus amiloride is optimal opinion; And consider from improving the quality of life, amlodipine plus telmisartan is prefered scheme. 